\title{
Rational targeted therapeutics for double-hit lymphoma
}

\author{
Nancy G Azizian¹,2, Yuanhui Liü1,2, Lan V Pham³ \& Yulin Li*,1,2 \\ ${ }^{1}$ Center for Immunotherapy Research, Houston Methodist Research Institute, Houston, TX 77030, USA \\ ${ }^{2}$ Department of Medicine, Weill Cornell Medical College, New York, NY 10065, USA \\ ${ }^{3}$ Department of Hematopathology, Division of Pathology \& Laboratory Medicine, The University of Texas MD Anderson Cancer \\ Center, Houston, TX 77030, USA \\ *Author for correspondence: yli@houstonmethodist.org
}

"Due to simultaneous activation of these driver oncogenes, DHLs are among the most aggressive and chemoresistant lymphoma subtypes with minimal treatment options"

\begin{abstract}
“Double-hit lymphomas with MYC and BCL2 translocations can be effectively treated by combined targeting of the driver oncogenes".
\end{abstract}

First draft submitted: 21 August 2019; Accepted for publication: 27 August 2019; Published online: 5 December 2019

Keywords: BCL2 • combination therapy $\bullet$ DHL $\bullet$ MYC • XPO1

Double-hit lymphomas (DHL) are a heterogeneous group of high-grade B-cell lymphomas with translocations involving $M Y C, B C L 2$ and/or BCL6 [1]. Due to simultaneous activation of these driver oncogenes, DHLs are among the most aggressive and chemoresistant lymphoma subtypes with minimal treatment options. The standard RCHOP (rituximab, cyclophosphamide, doxorubicin, vincristine and prednisone) combination treatment cures only a small fraction of the patients and most of the treated patients will experience disease relapse and progression. More intensive regiments, such as DA-EPOCH-R (dose-adjusted etoposide, prednisone, vincristine, cyclophosphamide, doxorubicin and rituximab), while improving the clinical outcomes, may give rise to serious complications $[2,3]$. Treatment of DHL currently remains a highly unmet need and therefore, novel therapeutics with low toxicity should be developed and tested specifically for DHL patients.

Targeted therapeutics is an attractive approach for the treatment of DHLs, as it has the advantage of inducing tumor regression in the absence of systemic toxicities associated with conventional chemotherapies. As DHLs are commonly driven by $M Y C$ and $B C L 2$, an effective therapy requires the combined targeting of both oncogenes. In the past few years, several BCL2 inhibitors have been developed for clinical use, an example of which, ABT-199 (Venetoclax), has been approved by the US FDA (MD, USA) for the treatment of chronic lymphocytic leukemia. In contrast, the development of specific MYC inhibitors has proven challenging due to its biophysical properties as a transcription factor. Despite decade-long efforts toward the development of small molecule chemical inhibitors of MYC protein, its direct in vivo targeting has not been feasible, and $M Y C$ is still considered 'undruggable' [4]. In the absence of effective chemical inhibitors, an alternative strategy to eradicate $M Y C$-driven cancers is through a synthetic lethality approach. Targeting genetic vulnerabilities specific to $M Y C$ overexpressing cancer cells can selectively eradicate those cells, without affecting the normal, noncancerous populations. Multiple synthetic lethal targets of MYC have been thus far identified. Inhibition of candidate gene products, such as BRD4, CDK9, PLK1 and others [5], demonstrates synthetic lethality with activated $M Y C$ in various cancer contexts. Therefore, selective targeting of these gene products could be a viable alternative in the treatment of $M Y C$-driven tumors, including DHLs.

Our recent work has demonstrated that simultaneous targeting of $M Y C$ and $B C L 2$ driver oncogenes in DHL can be achieved through the combined use of XPO1 and BCL2 inhibitors. XPO1 is an export receptor involved in nuclear-cytoplasmic transport of protein and RNA cargoes. XPO1 has been shown to transport several tumor suppressor proteins, such as $\mathrm{p} 53$, p27, BRCA1 and IKB [6]. In addition, XPO1 forms a complex with the mRNA

Future Medicine 
cap-binding protein eukaryotic initiation factor $4 \mathrm{E}$ (eIF4E) to export multiple oncoprotein mRNAs (such as $M Y C$, BCL2 and PIM1), promoting the synthesis of oncoproteins [7]. Genomic amplification or mutation of XPO1 has been observed in several human cancers, including multiple myeloma, leukemia and lymphoma. The efficacy of XPO1 inhibitors in cancer treatment is currently being tested in clinical trials [8]. One of the XPO1 inhibitors, KPT330, was recently approved by FDA for the treatment of multiple myeloma. We have observed in a panel of DHL cell lines that XPO1 inhibition using KPT-330 and KPT-8602 abrogates MYC protein expression, inducing massive tumor cell apoptosis. Combined use of XPO1 and BCL2 inhibitors is highly synergistic in vitro in eradicating DHL cells. In a mouse model of DHL bearing primary patient-derived xenograft tumor cells, combined treatment with XPO1 and BCL2 inhibitors blocks tumor progression and extends host survival [9]. Of particular interest, we found that the animals treated with the drug combination exhibit significantly lower brain metastasis compared with other groups. Both DHL and Burkitt's lymphoma are known to spread to a patient's central nervous system [10] at a much higher rate than other lymphoma subtypes. Brain involvement in lymphoma patients generally confers a more abysmal prognosis with a median survival of several months [10]. Our study with human patient-derived xenograft tumors reveals that combined targeting of XPO1 and BCL2 blocks brain metastasis of DHL tumors. Thus, such combined targeted therapies may prove effective in preventing tumor spread to the brain. Our finding is in concordance with a recent case report, where the use of KPT-330, was shown to restrain the central nervous system relapse in one diffuse large B-cell lymphoma patient [11]. In this light, the role of XPO1 as a target in the prevention and treatment of brain metastasis of lymphoma deserves further preclinical investigation.

As shown in both animal models and human patients, the clinical benefits of targeted therapies are often shortlived. Despite initial massive tumor cell death and effective tumor debulking, resistance to targeted agents and tumor relapse occurs frequently [12]. Although combined targeted therapies, aimed at simultaneous suppression of driver oncogenes would be more effective than targeted therapy with single agents, the development of drug resistance and tumor relapse following the combination therapy is still expected. The engagement of immune system is therefore required to achieve a sustained and long-term clinical outcome.

Anti-CD20 immunotherapy functions by engaging the immune system via antibody-dependent cellular cytotoxicity, complement-dependent cytotoxicity and adaptive cellular immune responses [13]. The classical anti-CD20 antibody for human B-cell lymphoma, rituximab, has demonstrated long-lasting survival benefits across multiple lymphoma subtypes. However, DHL tumor cells often have markedly reduced CD20 expression [14,15], contributing to resistance to rituximab and poor survival. In addition, multiple FDA-approved targeted agents commonly used in the treatment of human lymphomas, including ibrutinib, lenalidomide, bortezomib and idelalisib, have been shown to suppress CD20 expression on tumor cells by various mechanisms [16-18]. Consequently, the use of these targeted agents may significantly impair the efficacy of anti-CD20 immunotherapy using rituximab and other CD20 antibodies, such as ofatumumab. Not all targeted agents, however, decrease the expression of CD20 on lymphoma cells. Inhibitors of histone deacetylases (HDAC), such as entinostat, romidepsin and valproic acid, are known to augment CD20 expression on lymphoma cell membrane and potentiate the efficacy of anti-CD20 antibodies [19]. Additionally, inhibitors of DNA methyltransferases, such as azacytidine and decitabine, can partially restore CD20 expression in CD20-negative lymphoma cells and potentially enhance the effects of anti-CD20 therapies [20]. Thus, development of strategies that utilize the synergistic combination of targeted and immunotherapeutic agents in the treatment of DHL is of immense importance.

Combined targeting of $M Y C$ and BCL2 driver oncogenes, coupled with activation of immune response may serve as the therapy of choice culminating in a durable clinical response. As the first step, the potential synergistic or antagonistic effects of drug combinations can be screened in vitro using a diverse panel of DHL tumor cells. Subsequently, these candidate drug combinations should be tested in vivo in animal models with a functional immune system, including humanized or immunocompetent syngeneic mice, prior to clinical trials in DHL patients. In this light, combining targeted and immune therapies for DHL treatment, will provide a better means for effective tumor regression and immune system activation, achieving long-term survival benefits without significant toxicity.

Financial \& competing interests disclosure

The authors have declared no conflict of interests. Y Li is funded by a Career Transition Award from NIH (K22CA207598). The authors have no other relevant affiliations or financial involvement with any organization or entity with a financial interest in or financial conflict with the subject matter or materials discussed in the manuscript apart from those disclosed.

No writing assistance was utilized in the production of this manuscript. 


\section{Open access}

This work is licensed under the Attribution-NonCommercial-NoDerivatives 4.0 Unported License. To view a copy of this license, visit http://creativecommons.org/licenses/by-nc-nd/4.0/

\section{References}

1. Swerdlow SH, Campo E, Pileri SA et al. The 2016 revision of the World Health Organization classification of lymphoid neoplasms. Blood 127(20), 2375-2390 (2016).

2. Oki Y, Noorani M, Lin P et al. Double hit lymphoma: the MD Anderson Cancer Center clinical experience. Br. J. Haematol. 166(6), 891-901 (2014).

3. Dunleavy K, Fanale MA, Abramson JS et al. Dose-adjusted EPOCH-R (etoposide, prednisone, vincristine, cyclophosphamide, doxorubicin, and rituximab) in untreated aggressive diffuse large B-cell lymphoma with MYC rearrangement: a prospective, multicentre, single-arm Phase II study. Lancet Haematol. 5(12), e609-e617 (2018).

4. Mckeown MR, Bradner JE. Therapeutic strategies to inhibit MYC. Cold Spring Harb. Perspect. Med. 4(10), a014266 (2014).

5. Tan J, Li Z, Lee PL et al. PDK1 signaling toward PLK1-MYC activation confers oncogenic transformation, tumor-initiating cell activation, and resistance to mTOR-targeted therapy. Cancer Discov. 3(10), 1156-1171 (2013).

6. Gandhi UH, Senapedis W, Baloglu E et al. Clinical implications of targeting XPO1-mediated nuclear export in multiple myeloma. Clin. Lymphoma Myeloma Leuk. 18(5), 335-345 (2018).

7. Topisirovic I, Siddiqui N, Lapointe VL et al. Molecular dissection of the eukaryotic initiation factor 4E (eIF4E) export-competent RNP. EMBO J. 28(8), 1087-1098 (2009).

8. Garzon R, Savona M, Baz R et al. A Phase I clinical trial of single-agent selinexor in acute myeloid leukemia. Blood 129(24), 3165-3174 (2017).

9. Liu Yuanhui, Azizian NG, Dou Yaling, Pham Lan V, Li Yulin. Simultaneous targeting of XPO1 and BCL2 as an effective treatment strategy for double-hit lymphoma. J Hematol Oncol (2019) doi:10.1101/688093 (Epub ahead of print).

10. Qualls D, Abramson JS. Advances in risk assessment and prophylaxis for central nervous system relapse in diffuse large B-cell lymphoma. Haematologica 104(1), 25-34 (2019).

11. Bobillo S, Abrisqueta P, Carpio C et al. Promising activity of selinexor in the treatment of a patient with refractory diffuse large B-cell lymphoma and central nervous system involvement. Haematologica 103(2), e92-e93 (2018).

12. Sawyers C. Targeted cancer therapy. Nature 432(7015), 294-297 (2004).

13. Abes R, Gelize E, Fridman WH, Teillaud JL. Long-lasting antitumor protection by anti-CD20 antibody through cellular immune response. Blood 116(6), 926-934 (2010).

14. Pham LV, Lu G, Tamayo AT et al. Establishment and characterization of a novel MYC/BCL2 'double-hit' diffuse large B cell lymphoma cell line, RC. J. Hematol. Oncol. 8, 121 (2015).

15. Wu D, Wood BL, Dorer R, Fromm JR. 'Double-Hit' mature B-cell lymphomas show a common immunophenotype by flow cytometry that includes decreased CD20 expression. Am. J. Clin. Pathol. 134(2), 258-265 (2010).

16. Skarzynski M, Niemann CU, Lee YS et al. Interactions between Ibrutinib and anti-CD20 antibodies: competing effects on the outcome of combination therapy. Clin. Cancer Res. 22(1), 86-95 (2016).

17. Bil J, Winiarska M, Nowis D et al. Bortezomib modulates surface CD20 in B-cell malignancies and affects rituximab-mediated complement-dependent cytotoxicity. Blood 115(18), 3745-3755 (2010).

18. Bojarczuk K, Siernicka M, Dwojak M et al. B-cell receptor pathway inhibitors affect CD20 levels and impair antitumor activity of anti-CD20 monoclonal antibodies. Leukemia 28(5), 1163-1167 (2014).

19. Shimizu R, Kikuchi J, Wada T, Ozawa K, Kano Y, Furukawa Y. HDAC inhibitors augment cytotoxic activity of rituximab by upregulating CD20 expression on lymphoma cells. Leukemia 24(10), 1760-1768 (2010).

20. Hiraga J, Tomita A, Suzuki N, Takagi Y, Narita M, Kagami Y. Partial restoration of CD20 protein expression and rituximab sensitivity after treatment with azacitidine in CD20-negative transformed diffuse large B cell lymphoma after using rituximab. Ann. Hematol. 97(11), 2253-2255 (2018). 